\title{
Foetal Developmental Origins of Adult Onset Non-Insulin Dependent Diabetes Mellitus (NIDDM)
}

Sophie Stephenson and Adam Cunliffe*

Department of Applied Sciences, London South Bank University, London SE10AA, United Kingdom

\begin{abstract}
There is a global non-insulin dependent diabetes mellitus epidemic, and through understanding the foetal origins of the disease it may become possible to impact the development of the disease. During pregnancy a foetus is entirely dependent on nutrients supplied by the mother via the placenta, meaning alterations to the composition or quantity of maternal nutrition can lead to infants being born with low birth weights. Low birth weight is classified as a weight at birth of $\leq 2.5 \mathrm{~kg}$ by the World Health Organisation. In $1990 \mathrm{Dr}$. David Barker published the "foetal origins of adult disease" hypothesis in order to explain the observed epidemiological relationship between birth-weight and future disease development. It is now understood that alterations to gene expression occurring during foetal development, can then go on to increase the risk developing chronic non-communicable diseases, such as noninsulin dependent diabetes mellitus (NIDDM) in adulthood. However, the exact mechanisms through which this takes place remains unclear. The events during development which result in diminished foetal growth are multifactorial. A foetus's maximal growth potential is determined genetically, however maternal nutrition both pre and post conception is capable of influencing growth trajectory. This review will examine the existing body of evidence regarding the role of adaptive changes occurring during foetal development in relation to the subsequent development of NIDDM in adulthood. As well as considering the potential mechanisms through which these events could be mediated.
\end{abstract}

Keywords: Pregnancy; Maternal nutrition; Cardiovascular disease; Nutrients supplied; Nutritional status

\section{Introduction}

The foetal origins of adult disease hypothesis (FOAD hypothesis) was first proposed by Dr. D J Barker in 1990 in order to explain the observed epidemiological relationship between weight at birth and relative risk of disease development later in life [1]. In his original paper Barker discussed evidence that suggested events occurring during foetal development are capable of influencing the risk of developing a chronic degenerative disease in adulthood. He hypothesised that factors such as poor maternal nutrition both prior to conception and during pregnancy could lead to a low birth-weight. And that low birth-weight was indicative of risk for going on to develop disease in adulthood. The foetal and infant origins of adult disease paper focused on the infant developmental origins of cardiovascular disease and strokes. Subsequently it has been ascertained, through epidemiological data, that babies born with low birth-weights are more likely to develop a number of chronic diseases in adulthood. This includes cardio vascular disorders (CVD), hypertension and non-insulin dependent diabetes mellitus (NIDDM). Prior to the development of the FOAD hypothesis it was thought that chronic non-communicable disease in adulthood were the result of events occurring during adult life combined with predetermined genetic susceptibility to such diseases.

Nutrition represents a major modifiable factor in a person's life for preventing disease development and promoting overall health and wellbeing. During development a foetus is entirely dependent on the nutrients supplied by the mother via the placenta and the umbilical cord. It is now known that birth-weight is capable of functioning as a proxy for foetal nutritional status during gestation. Alterations to the quantity or composition of maternal nutrition can have profound and far reaching consequences for the foetus, resulting in physiological changes which can act to alter the relative risk of disease development. Low birth-weight in this instance classified as a weight at birth of less than $2.5 \mathrm{~kg}$ by the World Health Organisation (WHO) [2]. Weight at birth not only indicates nutritional supply during development, but also suggests the foetuses' growth trajectory in adult life.
Some of the most cited epidemiological studies in this area include the Hertfordshire cohort, the Helsinki study and the Dutch famine. These studies have established the importance of good maternal nutritional status during pregnancy and the importance of understanding the relationship between maternal nutrition and the weight at birth of their offspring.

The Hertfordshire Cohort comprised of 15,000 participants born between 1911 and 1930. The participants were weighed at birth then again at 1 year old. In both male and female participants, it was found that individuals weighing less than or equal to $2.5 \mathrm{~kg}$ at birth were more likely to have developed impaired glucose tolerance or NIDDM by age 64 years [3].

The Helsinki Birth Cohort studied around 13,000 participants born from 1934 to 1944 [4]. The study tracked growth and development of participants using a number of different anthropological measures taken from birth to 11 years, with a trace rate of $92 \%$ [4]. It was observed that participants with low Body mass index (BMI) at birth and at 2 years but a high BMI at 11 years were more likely to development insulin resistance by age 62 years [4]. The Helsinki Birth Cohort gathered measurements from participants up to 11 years of age whereas the Hertfordshire Cohort took measurements at birth and at 1 year, increasing the reliability data gathered by the Finnish researchers.

*Corresponding author: Adam Cunliffe, Associate Professor, Department of Applied Sciences, London South Bank University, 103 Borough Road, London, SE1 OAA, United Kingdom, Tel: 078145821723; E-mail: cunliffa@Isbu.ac.uk

Received September 07, 2018; Accepted October 10, 2018; Published October 18 2018

Citation: Stephenson S, Cunliffe A (2018) Foetal Developmental Origins of Adult Onset Non-Insulin Dependent Diabetes Mellitus (NIDDM). J Nutr Food Sci 8: 733 doi: 10.4172/2155-9600.1000733

Copyright: (c) 2018 Stephenson S, et al. This is an open-access article distributed under the terms of the Creative Commons Attribution License, which permits unrestricted use, distribution, and reproduction in any medium, provided the original author and source are credited. 
The Dutch famine took place between December 1944 and April 1945, during which regions of the Netherlands were occupied by the German Nazi soldiers during World War 2. During the Nazi occupation daily rations fell from approximately 1800 calories in December 1943 to below 1000 calories in November 1944. At the famines peak, daily rations fell as low as $400-800$ calories a day [5]. A number of studies have examined the long-term consequences of the Dutch famine. In one particular study 2414 singleton births between November 1943 to February 1947 were examined when participants were aged between 50-58 years. An individual was considered exposed to the famine if the average daily rations fell below 1000 calories for any consecutive 13-week period during gestation [5]. It was found that exposure to undernutrition at any stage of development in utero led to reduced glucose tolerance and raised insulin concentrations when compared to individuals who were not exposed to famine in utero. These finding are in line with the those of the Hertfordshire cohort and the Helsinki study. All of which support the FOAD hypothesis.

There are a number of explanatory models which can be used when describing the observed association between birth-weight and subsequent disease development. Many of these models centre around the fact that there are periods during foetal development in which the foetus is sensitive to external stressors, such as nutrient availability. This includes 'developmental plasticity', this is a genotype's capacity to produce alternative forms of structure, physiological state or behaviour in response to environmental conditions. In the past, the ability to elicit phenotypic and morphological alteration during development was beneficial, aiming to increase an organism's probability of survival to sexual maturity and successfully carrying on the gene line. The adverse environment experienced in utero leads to phenotypic changes aiming to increase energy conservation, to ultimately maximise the individual's probability of survival, this is known as the "thrifty phenotype" [6]. The thrifty phenotype is advantageous under conditions poor in nutrition, resulting in increased peripheral glucose utilisation which would have be historically advantageous. But can be disadvantageous in an environment where food is abundant. There is also thought to be some epigenetic component involved in inducing phenotypic adaptations. Epigenetics refers changes in gene expression occurring at the level above the DNA, inheritable changes to the phenotype, or to the way in which a gene is expressed.

The "Predicted Adaptive Response" hypothesis suggests that the environment in utero is involved in "programming" the foetus in preparation for a similar postnatal environment e.g. one sparse in nutrition [7]. There are periods during foetal development in which the foetus is sensitive to external environmental pressures. The mechanisms resulting in low birth-weight infants are also thought to be involved with the alterations to foetal programming that lead to the increased likelihood of these individuals developing chronic illness, such as NIDDM, in adult life.

An estimated $15.5 \%$ of all births globally are thought to be low birth-weight, with $95.6 \%$ of these occurring in less economically developed countries [2]. With India being country the with the highest incidence of infants born with low birth-weight per capita [8].

As has been demonstrated by numerous scientific studies low birth-weight represents a risk factor for the future development of a number of chronic disorders, including NIDDM. Diabetes mellitus can be described as an organism-wide inability to utilize blood glucose due to disturbances pertaining to insulin production and/ or function. NIDDM is a preventable disorder which typically manifests in adulthood, aged 30 years and over, and can be characterised as a loss of insulin sensitivity. According to the WHO the number of incidences of NIDDM is increasing globally, with the number rising from 108 million in 1980 to 422 million in 2014 [9]. In 2015 there was an estimated 1.6 million deaths as a result of diabetes, and it can play a major contributing role in the development of blindness, heart disease and lower limb amputation. Projections made by the WHO claim that diabetes will be the $7^{\text {th }}$ leading cause of death globally by 2030 . The treatment and management of NIDDM represents a huge burden on the current healthcare infrastructure. However, through greater understanding of the foetal developmental origins of NIDDM it may be possible to identify at risk individuals and establish targeted public health campaigns aimed at preventing the onset of NIDDM.

The FOAD hypothesis is used to explain an epidemiological relationship between low birth-weight and disease development later in life. The theory is generally widely accepted within the world of developmental genetics and the wider scientific community. However, the present understanding fails to discern the exact mechanisms through which the events occurring in utero go on to increase an individual's risk of disease development.

\section{Aims and Hypothesis}

The aim of this review is to examine the existing body of evidence regarding the role of adaptive changes occurring during foetal development in relation to the development of NIDDM in adulthood. With particular emphasis placed on exploring the proposed mechanisms leading to mature onset NIDDM as a result of epigenetic changes. As well as proposing future directions for research based on gaps in the current understanding. In order to achieve this, concepts such as the FOAD hypothesis, Predicted Adaptive Response hypothesis and epigenetic influence will be critically reviewed. For the purpose of this review only the developmental origins of NIDDM will be discussed.

\section{Discussion}

\section{Factors capable of influencing foetal growth during pregnancy}

It is known that a number of different factors are capable of influencing foetal growth patterns during pregnancy and development. The maximal foetal potential growth trajectory is determined genetically and regulated by a number of genes, inherited both maternally and paternally. Though the foetal genome is responsible for the growth potential, it is in fact the nutritional and hormonal environment in which the foetus develops which ultimately determines size at birth [10]. During development foetuses are entirely dependent on nutrients supplied by the mother via the placenta and the umbilical cord [11]. There are a number of potential ways in which foetal growth can be restricted such as; morning sickness, placenta size, functional capacity of nutrient transfer between the mother and placenta and socioeconomic factors.

Morning sickness or nausea and vomiting of pregnancy (NVP) is one such instance where the nutritional intake of the foetus may be affected during pregnancy. It is estimated that between $50 \%-90 \%$ of women experience NVP during the first 12 weeks of pregnancy [12]. With $0.5 \%-3 \%$ of those individuals experiencing a more severe form of NVP known as hyperemesis gravidarum (HP), which is characterised by severe nausea, vomiting, dehydration and in some cases hospitalisation [12]. One study examined the epidemiology of NVP through studying the pregnancy outcomes of 356 women. Where it was found that women of Caucasian background were more likely to experience NVP when compared to women of Black or Asian background [12]. In most cases NVP is experienced in the first trimester, with one $1.3 \%$ reporting 
NVP in the second trimester [12]. It is important to note that severe NVP is capable of eliciting a similar response to those induced when the mother is undergoing starvation [5]. And a number of animal studies performed in rodent populations have examined the role of nutrition in early pregnancy $[13,14]$. Both studies found that a poor nutritional intake during very early pregnancy lead to the activations of mechanistic pathways aimed at improving postnatal fitness in the short term. However, these adaptations could prove detrimental to health in the long term. Meaning individuals experiencing severe NVP in early gestation may be at risk of altering foetal growth trajectory even in very early stage pregnancy. The exact aetiology of NVP remains largely unknown and further investigation is required in this area. In order to establish contributing factors, severity of symptoms and the extent to which NVP is capable of influencing foetal growth patterns.

Social determinants of health such as socioeconomic class, income, access to healthcare and level of education all play a role in determining foetal nutritional status. With the greatest instance (95.6\%) of low birth-weights occurring in less economically developed countries, it is reasonable to suggest healthcare inequalities may play a contributing role in low birth-weight [2]. A number of studies have shown that there is an increased risk of low birth-weight when the mother is young ( $<18$ years) or older ( $>35$ years) at birth. Suggesting a "J-shaped curve" relationship between maternal age at birth and relative risk of low birth-weight [2]. In younger mothers the observed lower birth-weight may be due to lower socioeconomic standing, reduced income and reduced levels of education. As low levels of maternal education are a known risk factor for a child to be born with a low birth-weight [2]. In older mothers low birth-weight may be due to DNA abnormalities associated with the aging process [2].

It is possible to consider pregnancy in three separate compartments, the mother, the placenta and the foetus. Each of which with its own metabolism and capable of interacting with the others [15]. Placentas perform metabolic processes and therefore have their own energy requirements, and placental size and nutrient carrying capacity represent a limiting factor for overall foetal growth and birth-weight. Placenta size and functional capacity to perform nutrient transfer between the mother and foetus is critical for foetal growth and development. Placental abnormalities are a known risk factor for the development of gestational hypertension, which can result in a reduced uteroplacental flow, which in turn would lead to low birth-weight [2]. The initial development and establishment of the placenta occurs is very early pregnancy, as early as 8 days post conception, therefore maternal nutritional status in early pregnancy plays a role in placenta formation. This is in line with the experimental evidence gathered from studies carried out on rodents $[13,14]$. As placenta establishment occurs in very early pregnancy it is important to promote good nutritional status even prior to conception.

Observational epidemiological data suggests that the placenta also experiences "windows of sensitivity" during development, through doing so the placenta is capable of undergoing metabolic change in response to alterations in nutrient availability. It has been found that individuals who experience nutritional hardship during the third trimester gave birth to infants with decreased birthweight and placental weight [16]. Whereas individuals who experienced undernutrition in the first trimester experienced no alteration to birth weight but an increase in placental weight was observed [16]. Demonstrating that the placenta is capable of undergoing compensatory growth in the event that there is insufficient maternal nutrition during the initial stages of development, acting to circumvent the negative effects of foetal undernutrition [16]. Indicating the placenta is sensitive to external stressors throughout gestation, but particularly during later stage gestation when foetal nutritional demands reach their peak. In the past the exact extent to which the placenta is capable of influencing foetal growth and foetal phenotypic adaptations has been overlooked, and the crucial role of the placenta should be explored more thoroughly.

As discussed, the events leading to diminished foetal growth during pregnancy are multifactorial. The FOAD hypothesis has been criticised because factors other than nutrition are capable of causing a low weight at birth, such as hypoxia and stress. A foetus's maximal growth potential is dictated through genetic inheritance; however, it is thought that maternal nutrition during development is equally or more so important in dictating the actual weight of the offspring at birth. Factors such as NVP, socio-economic principles and nutrient transfer capacity of the placenta are all known to play a role in determining foetal growth.

\section{The role of preconceptional nutritional status}

As has been previously established, the quantity and composition of maternal nutritional intake during pregnancy has the ability to profoundly alter relative disease risk in the offspring. However, it is now known that it is not solely nutritional intake during pregnancy which is capable of influencing foetal growth, increasingly the role of preconceptional nutrition is being discussed. It is thought that there is a positive correlation between quality of preconceptional nutrition and the overall growth trajectory of the foetus. Current research suggests that preconceptional maternal nutrition is capable of creating epigenetic changes to the DNA which could then be passed onto offspring [5].

A number of studies involving animal populations have demonstrated the importance of preimplantation maternal nutrition $[13,14]$. In both experiments, the subjects were fed a diminished protein diet for the duration of the preimplantation phase and were then returned to a control diet for the remainder of the pregnancy. Implantation occurs when the zygote adheres to the wall of the uterus, in humans this typically occurs at about 8 days post conception. Through studying the effects of a low protein diet during the preimplantation period of rodent development, both Watkins and Kwong found that that this led to the activations of mechanistic pathways aimed at improving postnatal fitness in the short term. However, these adaptations could prove detrimental to survival in the long term $[13,14]$.

Another animal study performed in sheep demonstrated that ewes with poor nutritional status at conception and high nutritional intake during early stage pregnancy had increased placenta size. Whereas in ewes with good nutritional status at conception and high nutritional intake during early pregnancy smaller placentas were observed [17]. It is known that Placenta establishment occurs in very early pregnancy and its size may be reflective of maternal nutritional status at the time of conception [11]. The size of the placenta is able to function as an indirect measure of its capacity for nutrient transfer, through alterations to the surface area, therefore the larger the placenta the greater its capacity for nutrient transfer would be. Failure to establish a sufficient maternal-placental-foetal unit may lead to the nutritional requirements of the developing foetus to not be met [10].

Pregnancy is a hugely anabolic process, and tremendously energy expensive; the total energy cost of pregnancy is predicted to be around $85,000 \mathrm{kcal}$ or 300 calories/day [18]. Meaning individuals with poor preconceptional nutrition would lack the ideal levels of stored 
nutrients prior to conception and may be at risk of giving birth to low birth-weight children. Specifically, individuals living in poorer socioeconomic classes may be more at risk of experiencing inadequate calorie or micronutrient intake during the preconceptional period. Those living in less economically developed countries are also less likely to have access to educational resources about healthy lifestyles.

Through developing and promoting public health campaigns aimed at improving overall nutritional status of reproductive aged women it would be possible to improve the health span of potential offspring. Potentially targeting women from poorer socio-economic backgrounds who are the most at risk of poor nutrition. Current understanding acknowledges the importance of preconceptional nutritional status and through ensuring adequate nutritional status for women from a young age it would be possible to promote good health for the offspring in adult life. Most individuals do not know they are pregnant until 6-8 weeks into gestation, after which point implantation has already occurred. Therefore, it would be reasonable to expect their original preconceptional nutritional habits to persist up until this point. Highlighting once again the importance of good nutritional status and education in the preconceptional period.

\section{Intergenerational effects}

As previously discussed, current research suggests that maternal nutrition is capable of inducing epigenetic changes to the DNA, even prior to conception, which could then be passed onto offspring [5]. It has been found that those who were exposed to the Dutch famine during the first two trimesters of their own foetal development gave birth to lower weight babies when compared with women who were not exposed to famine during their own development [19]. Evidence also suggests that mothers with a low birth-weight have a tendency to give birth to thin offspring, providing evidence of a relation between maternal birth-weight and foetal weight at birth. Suggesting some level of inheritability of the short-term adaptations which occur during foetal develop if they are then able to be passed onto subsequent generations.

\section{Point in gestation restriction occurs}

It is known that pregnancy represents a time of rapid cell growth and development and good maternal nutrition is vital at every point. However, there are critical periods of time during development in which the foetus is sensitive to their external environment. It is unknown as to the exact point during development these "windows of sensitivity" occur, for how long they last and to what degree events occurring during this time are capable of influencing adult disease development. Through observing disease development in individuals exposed to nutritional distress at different points during development, it may be possible to develop some understanding as to the impact on nutrient deprivation at each point during development. During periods of growth and development an organism is sensitive to external stressors, and they are capable of producing permanent phenotypic adaptations as a response. Phenotypic adaptations aim to increase an organism's probability of survival to sexual maturity and successfully carrying on the gene line [20].

As has been previously discussed pregnancy is extremely energy expensive. With the nutritional demands of the foetus reaching their peak during the third trimester of pregnancy. Due to this increase in nutritional demand by the foetus weight at birth is most affected when exposed to alterations in maternal nutritional status during the third trimester [15]. During this period if the rapidly developing foetuses' nutritional requirements are not met there may lead to a number of metabolic alterations in order to compact the changes experienced in nutritional availability [10]. Which may include adaptations such as foetal wasting and oxidation of foetal amino acids [10]. There is experimental data to suggest that in the second half of gestation, foetal growth is controlled by both maternal and placental factors.

Results from research conducted following the events of the Dutch famine have shown that individuals exposed to maternal nutritional intake of below 1000 calories for at least 13 weeks at any point during gestation had increased insulin and glucose concentration. Irrespective of the point in gestation in which caloric restriction occurred [5]. Suggesting that mechanisms involved in the regulation of insulin and glucose concentrations are capable of undergoing alteration at any stage of development. However, another study found that impaired glucose tolerance was most prevalent in the offspring of women who were exposed to the famine only in late gestation [11].

Rodent studies have demonstrated that maternal nutritional status is capable of influencing embryonic development, even in very early pregnancy $[13,14]$. The Kwong research group found, that rats who were fed a low protein diet during the preimplantation period displayed reduced rates of cellular proliferation. Along with irreversible physiological changes as a result of the low protein diet during preimplantation. Severe NVP is common in early pregnancy, and it is known that this is capable of stimulating similar response pathways as those activated when a mother is undergoing starvation [5].

As established in the original FOAD hypothesis put forward by Barker in 1990, alteration to the quantity or quality of maternal nutrition can lead to a reduction in weight at birth. This theory is well established and generally accepted within the scientific community, however the exact time frame during development in which the foetus is particularly sensitive to the external environment has not been established. Evidence gathered from landmark studies such as the Dutch famine are able to provide some insight as to the effect of nutrient restriction at various point during foetal development. However, aspects such as timing, nature and degree to which there is maternal undernutrition act to influence the exact nature of subsequent disease development require further examination.

\section{Macronutrients}

As has previously been discussed, it is known that reductions in the overall quantity of maternal nutrition is able to cause alterations to foetal gene expression. Which can ultimately alter relative disease risk of chronic non-communicable diseases, such as NIDDM, later in life. The original FOAD hypothesis discussed the role of maternal undernutrition as a whole, rather than the specific composition of maternal diet during gestation. More recently the role of macronutrients in foetal development has undergone some investigation. Macronutrients are large organic compounds which can be utilised by organism as an energy source. Dietary macronutrients include protein, fat and carbohydrates. Glucose, amino acids and fatty acids represent the most important nutrients in foetal development [15].

Carbohydrates generally represent between $40 \%-75 \%$ of adult dietary intake. Carbohydrates are considered non-essential macronutrients, as the liver is capable of endogenous glucose production. However, carbohydrates are a prominent feature in most cuisine the world over as they are able to increase the palatability of food as well as acting as a transport vehicle for a number of micronutrients. Glucose constitutes the major respirative substrate for oxidative metabolism during foetal development, as glucose is able to pass via 
facilitated diffusion from the maternal bloodstream into the placenta [11]. Given this, it would be logical to suggest that increased maternal consumption of carbohydrates during gestation would act to increase foetal growth. However, one study involving 538 pregnant women who delivered to term, measured carbohydrate intake within the sample population [21]. It was found that individuals with high carbohydrate intake during early pregnancy gave birth to offspring with lower birthweights and reduced placenta mass. This was found to be independent of the mother's height and body mass index (BMI) during gestation.

Protein is an essential dietary macronutrient and is involved in the growth and repair of tissue, the production of enzymes, growth factors and hormones and are capable of providing energy. Multiple studies have cited the role of amino acids as an integral component in foetal development, including setting the level of protein requirement. Amino acids also become a major substrate for energy production [22]. It has been demonstrated experimentally, that rodents whose mothers had a low ratio of dietary protein during early pregnancy then went on to develop permanently altered hepatic glucose production and utilisation [13,14]. Suggesting the importance of adequate protein intake during gestation, and its role in regulation of hepatic glucose production and utilisation. Kwong's study in rats found that embryos of rodents fed low protein diet demonstrated slower rates of cellular proliferation as opposed to increased rates of cellular apoptosis, as was initially assumed by the authors. A study by Godfrey et al., measured dietary protein and dairy intake of 538 pregnant women who delivered to term. It was reported that individuals with low intake of dairy and meat were associated with decreased birth-weight and lower placenta weight. Again, independently of the mother's height and BMI during gestation. Individuals following a strict vegetarian or vegan diet do not consume meat and dairy products, which could potentially lead to reduced placenta size and lower birth-weight offspring. This is in line with epidemiological data, India has the highest global incidence of low birth-weight while also having the largest vegetarian population globally. Indicating the need for further research into the exact role of animal protein and dairy in foetal development.

Beta cells are cells found in the pancreas and are capable of the production and storage of insulin. Beta cells are capable of detecting circulating nutrient levels in adults there may be a similar mechanism in place during foetal development stages [22]. Current research understanding suggesting that amino acids are involved in the growth and development of beta cells [22].

It is known that size at birth is capable of functioning as a proxy for foetal nutrition during development. During development, glucose functions as the primary reparative substrate for oxidative metabolism and enters the placenta via facilitated diffusion [11]. However, experimental evidence suggest high carbohydrate intake during gestation may in fact result in impaired foetal growth. Protein is an essential macronutrient and reduced protein in rodents' intake during the preimplantation phase can result in phenotypic adaptations leading to altered glucose production and usage. In humans it is known that reduced protein intake during gestation can result in low birth-weight. Suggesting that those following a strict vegetarian or vegan diet may be more at risk of giving birth to offspring with low birth-weights.

\section{Micronutrients}

In the past, it is mainly the role of maternal nutrition as a whole which has been considered when discussing foetal nutrition and development. The potential role of micronutrients in foetal growth and development has been greatly overlooked. Micronutrients are organic compounds, vitamins, or inorganic elements, minerals, which play an essential role in promoting and maintaining general health and wellbeing. Micronutrients perform this role through acting as cofactors of enzymes involved in chemical reactions in the body and are also capable of acting as antioxidants. It is known that micronutrient deficiencies during pregnancy are associated with negative outcomes for example, folate deficiencies during gestation can cause neural tube defects, such as spina bifida, in offspring. However, there is a lack of good quality experimental data regarding the various roles of other micronutrients during foetal development, especially as to their role in not only foetal development but in long term health remains largely unknown. Maternal undernutrition is conventionally thought of as insufficient macronutrients intake.

To date there has been some work conducted as to the role of iron, vitamin $A$ and a number of $B$ vitamins.

Iron is an essential trace mineral and, in adults, is involved primarily in oxygen transport. Iron also plays a role in normal functioning of the immune system, especially in lymphocytes. During pregnancy there is an increase in iron requirement, due to increased maternal production of red blood cells required, not only the foetus, but also the placenta. Maternal deficiency has been associated with preterm delivery and low birth-weight [23]. Haem iron is considered the best dietary source of iron, found in meat and other animal products. Those who are not consuming haem iron from meat sources must consume non-haem iron found in plant sources. The iron absorbed from non-haem sources is not as readily absorbed as haem iron and has a reduced bioavailability. It is estimated that haem iron is absorbed 15\%-35\% better than iron from non-haem sources [24]. Those living in a low socioeconomic class may have reduced access to quality haem iron from animal sources and may be at risk of deficiency. Though supplementation may cause constipation, which is already a problem for many during pregnancy. One study examined the effects of iron supplementation during pregnancy, where 275 non-anaemic iron replete women less than 20 weeks pregnant were prescribed either iron tablets or a placebo, up until they reached 28 weeks gestation. This study found that the offspring of women who took iron supplements were significantly heavier and significantly less likely to be born with low birth-weight when compared to infants born to women receiving the placebo supplement [23]. Iron absorption can be inhibited by high non-starch polysaccharides NSP (fibre) intake. Meaning those with high dietary fibre intakes such as individuals following strict vegetarian or vegan diets may not only be at risk of reduced iron absorption. But they would also only be ingesting the less bioavailable non-haem iron from plant sources. India has the largest population of vegetarians globally, they also have the highest incidence of low birth-weight. Iron deficiency is the most common micronutrient deficiency globally and can lead to deficiency disorders such as iron deficiency anaemia. Anaemia is significantly associated with low birth-weight babies; therefore, it may be advisable to recommend supplementation of vitamins and minerals in order to prevent gestational anaemia. Anaemia observed during pregnancy can also be the result of an increase in overall fluid in the body and the red blood cell count remains the same.

Vitamin A is a group of organic retinoids which includes; retinol, retinal and retinoic acid. The roles of vitamin $\mathrm{A}$ in the adult body include the normal functioning of the immune system and in the maintenance of vision and eye health. Vitamin A is also involved in gene transcription and there is some evidence as to the role of vitamin $\mathrm{A}$ in the formation of pancreatic beta cells [25]. The evidence as to the role of vitamin A in foetal development is limited, and few studies have 
been performed as to the role of vitamin A in pancreatic development in human subjects. One rodent study has shown that low to moderate vitamin A deficiency both pre and post conception can lead to a reduction in both pancreatic beta cell number and area by up to $50 \%$ [25]. The reduced area and number of pancreatic beta cells observed by Matthews may have been due to a decrease in the level of beta cell formation in the test subjects or an increased level of cell apoptosis [25]. Reduced number and functional capacity of pancreatic beta cells is also observed in humans who are born at a low birth-weight. This work suggests the possible role of vitamin A in humans leading to the same deficiency in beta cells also observed in humans born with low birthweights. Vitamin A is involved in gene transcription, cell proliferation and differentiation in a range of cell lines, this could be its potential role in pancreatic beta cell production [25]. Vitamin A deficiency is common in communities where rice represents the main calorie source, due to the fact that rice is lacking in beta carotene or provitamin A. Through developing intervention programs targeting individuals in these communities it would be possible to improve pregnancy outcome and the over health of subsequent generations.

$B$ vitamins are a group of water soluble organic molecules involved in growth, development and differentiation. This class of vitamins includes B9 (folate), B12 (cobalamin), B6 (Pyridoxal phosphate) and B2 (Riboflavin). A number of B vitamins also play a role in DNA synthesis and post transcriptional modifications to proteins. Vitamins B9, B6 and B12 are also involved in the metabolism of homocysteine into methionine [26]. Disturbances in the metabolism of any of these compounds results in increased circulating levels of homocysteine, plasma homocysteine concentration is inversely related to vitamin B12 levels. One study has examined the relationship between maternal levels of homocysteine, vitamin B12 and folate and infants weight at birth [8]. It was found that infants who were small for gestational age were born to smaller mothers who had higher circulating levels of homocysteine, when compared with mothers who gave birth to average for gestational age babies [8]. Low circulating levels of vitamin B12 throughout gestation was associated with a significantly higher risk of infant growth restriction [8]. It was found that even by the age of 6 years children born to mothers with high circulating levels of folate but low levels of circulating B12 had the highest level of insulin resistance when compared to others in the same study [8]. Those following a strict vegetarian or vegan diet are more at risk of developing a vitamin $\mathrm{B} 12$ deficiency, due to the fact that B12 is almost exclusively found in products of animal origin. Women from poor socioeconomic backgrounds are more likely to be at risk of B vitamin deficiencies, especially B12, this acts to create a health inequity. Folate and B12 are both carbon 1 metabolites, which may be capable of attaching to DNA molecules are altering gene expression and may be involved in epigenetic changes occurring above the level of the DNA leading to alterations in gene expression [27].

Other essential minerals such as magnesium and zinc are known to play roles in insulin sensitivity, secretion and storage in the adult body [27]. However, their potential role in foetal development remains unknown. Deficiencies in iron, zinc, folate or vitamin B12 during foetal development may lead to a reduction in the number of pancreatic beta cells which is observed in individuals with a low weight at birth [27]. Suggesting once again that alterations to pancreatic beta cell development is not the result of one individual factor, but a number of contributing factors. In particular, altered zinc metabolism is known to be involved in the development of NIDDM, this may be due to reduction in the activity of insulin -like growth factors as a result of deficiencies, leading to diminished foetal growth [27].
The majority of experimental evidence regarding the role of micronutrients in foetal development has been derived from studies conducted on non-primate mammals i.e. rodents. While the conclusions drawn from these studies warrant further investigation as to the role of micronutrients in human foetal development, they themselves are not sufficient grounds upon which to base human maternal micronutrients recommendations for wider public health campaigns.

\section{Physiological alterations}

It is known that events occurring in utero are capable of inducing phenotypic adaptations. In order to increase fitness to the environment and improve the probability of offspring survival to sexual maturity and going on to reproduce in order to continue the gene line. Irrespective of the cause, reduced birth-weight is associated with alterations in function and development of the endocrine system. Adaptive changes occur in response to limited nutritional supply in order to prioritise growth differently. The changes to growth prioritisation are aimed at increasing short term survival which can lead to increased energy conservation in order to maximise the individual's probability of survival, this is known as the "thrifty phenotype" [6]. However, it is these alterations to endocrine function which, in the long-term, acts to increase risk profile for a number of non-communicable disorders, such as NIDDM.

Food supply is a major selective force in humans, in our evolutionary history those able to adapt to the relative abundance or scarcity of food were, in general, the most successful. When the foetal demand for nutrition cannot be met there may be alterations to the foetal programming in order to minimise negative repercussions [20]. However, if there is a change in the availability of nutrition in the postnatal environment this adaptation can prove to be dysfunctional [20]. It is this "mismatching" between the pre and postnatal environment which can lead to the increase in risk factors for the development NIDDM [20].

Poor in utero environments have been shown to result in a reduced number of pancreatic beta cells in both humans and rodents, which can lead to decreased insulin secretion $[10,28]$. Pancreatic beta cells are cells located in the pancreas, specifically inside the islets of Langerhans, which are capable of producing, storing and secreting the hormone insulin. Insulin is a hormone involved in the homeostatic regulation of blood sugar, through promoting the uptake of glucose at the cellular level and stimulating glycogen production at the level of the liver. Hepatic resistance to insulin has also been observed shortly after birth is small for gestational age babies [11]. However, it may not be solely quantitative deficiency in beta cells leading to reduced functional capacity, there may also be alterations to their function. One study has found that insulin secretion can be reduced by up to $30 \%$ in adults born with low birth-weights, which can ultimately lead to the development of NIDDM [6]. Reduction in beta cell function and smaller islets can lead to reduced functional capacity for insulin production [11]. It has been found that increased levels of circulating glucocorticoids are capable of leading to reduced rates of pancreatic beta cell formation [29]. Glucocorticoids are a class of steroid hormones, one example of a glucocorticoids being cortisol.

As previously discussed, glucose is the main substrate utilised by the foetus during development. When glucose availability is reduced there is some evidence to suggest that there will be alterations allowing for the foetus to become less dependent on the oxidation of glucose for energy. Instead utilising other substrates such as amino acids in order to fulfil their energy requirements [10]. When amino acids are 
being utilised as an oxidative substrate there are decreased amino acids demands for growth, leading to the birth of a baby small for gestational age. There is also some evidence to suggest abnormalities to foetal muscle structure and function stem from events occurring midgestation, such as maternal undernutrition, may lead to interferences with insulin's functional capacity to promote glucose uptake into muscle cells [10].

Rodent studies found that low protein diets during preimplantation leads to reduced rates of cellular proliferation, rather than increased rates of cellular apoptosis [14]. The beta cells of the islets of Langerhans grow rapidly during foetal development, in normal individuals $10-20 \%$ of the blood flow to the pancreas is supplied directly to the islets [22]. It would be expected that any alterations to the anatomical structure of the islet could contribute to changes in functional capacity. If low maternal protein intake leads to reduced rates of cellular proliferation it is expected that there would be a reduction in the number of beta cells.

Regardless of the cause of the reduced birth-weight there are associated alterations in the endocrine systems. Low birth-weight is a marker for further alterations in functions such as endocrine functions and development of glucose-insulin interactions and insulin-growth factor axes [11]. However, the mechanisms controlling organ development is poorly understood. It is evident that nutrient restrictions at any point during gestation is capable of triggering changes to endocrine development, suggesting that endocrine development maintains a certain level of plasticity throughout development.

\section{The role of catch up growth}

The royal college of paediatrics and child health $(\mathrm{RCPCH})$ manufacture and distribute standardised growth charts for use by registered healthcare professionals across the UK. The RCPCH's growth charts are used to assess growth in children relative to growth standards developed by the WHO. These are used in order to track growth in children from birth to 18 years. In medical professional fields, a small infant who then goes on to experience exponential postnatal growth is considered desirable. On the grounds that low birthweight infants are at a greater risk of infant mortality than those who are heavier at birth [30]. It is known that rapid postnatal weight gain in the short term is capable of improving an infant's expected health outcomes in terms of morbidity and mortality. However prolonged compensatory growth has the converse effect and can lead to adverse health outcomes in adult life. Evidence suggests that excessive catch up growth acts to increase the risk of development of a number of chronic diseases relative to those with higher birth-weight [3]. This includes disorders such as CVD, hypertension and NIDDM. The effect is most pronounced in individuals with low birth-weight who then undergo rapid catch-up growth during childhood. Who have been shown to be at an even greater risk of disease development when compared with individuals born with a low birth weight but did not undergo this period of compensatory growth.

A table detailing the odds ratios for the development of NIDDM based on birth-weight and body mass index (BMI) at age 11 years has been calculated by Dr Barker [3]. According to the growth charts provided by the RCPCH the median height and weight expected for an 11-year-old boy is $35 \mathrm{~kg}$ and $1.46 \mathrm{~m}$ respectively, giving them a BMI of $16.4 \mathrm{~kg} / \mathrm{m}^{2}$. Following the odds ratio table, an individual with a birth-weight of $3.0 \mathrm{~kg}$ who then at age 11 years had a BMI of $16.4 \mathrm{~kg} / \mathrm{m}^{2}$ has a probability of 1.3 for going on to develop NIDDM. This is compared to odds of 0.9 in favour of NIDDM development in an individual who was $4.0 \mathrm{~kg}$ at birth and the same BMI at age 11 years. These are just the odd ratios for individuals who have median height and weight at age 11 years. As shown by Barker individuals born weighing $3.0 \mathrm{~kg}$ who then go on to have a BMI of $>17.6 \mathrm{~kg} / \mathrm{m}^{2}$ are 2.5 times as likely to develop NIDDM in adulthood. A BMI of $\geq$ $17.6 \mathrm{~kg} / \mathrm{m}^{2}$ could be seen in a child weighing $\geq 38 \mathrm{~kg}$ which would be below the $75^{\text {th }}$ percentile for children age 11 years. Or alternatively a child with a height of $1.41 \mathrm{~m}$ at age 11 years, which is above the $25^{\text {th }}$ percentile for height at this age.

There are clear short-term health benefits associated with early catch up growth, centring around reductions in infant morbidity and mortality and reduction in the number of hospitalisations [31]. Nevertheless, excessive compensatory growth represents an indisputable risk factor for the development of chronic disease among individuals born with low birthweights. By failing to take weight at birth into consideration the RCPCH's growth charts are not able to accurately judge the health of a child and relative disease risk. Though there are well documented short-term benefits of catch up growth for low birth weight infants there is a definite trade off in terms of potential long-term health outcomes. Through the redevelopment of these standardised growth charts it might become possible to identify individuals with a low birth-weight and monitor their grown and relative disease risk. Aiming to reduce risk factors prior to the development of chronic conditions such as NIDDM.

\section{Nutritional habits later in life}

For thousands of years it has proved beneficial to have the capacity to predict the availability of nutrients in postnatal life while in utero. In the past the environment experienced in utero would have provided adequate information about the external environment, making the ability to adapt beneficial and therefore positively selected for [7]. The "Predicted Adaptive Response" hypothesis suggests that the environment in utero is involved in "programming" the foetus in preparation for a similar postnatal environment e.g. one sparse in nutrition [7].

Individuals who continue to experience a non-ideal nutritional climate in the postnatal environment have undergone phenotypic adaptations in order to effectively deal with this eventuality. As a result, they tend to have smaller insulin producing pancreatic beta cells, which would lead to a reduced functional capacity to produce insulin. Reduced insulin production would lead a diminished ability to clear glucose form the blood stream effectively, as has been demonstrated in rodent studies with reduced number of pancreatic beta cells. Adaptations leading to decreased beta cell function have also been shown to lead to increased glucose utilisation and reduced demand for amino acids [11]. During adult life, pancreatic beta cells have the capacity to detect the availability of circulating nutrients [22]. If there are fewer pancreatic beta cells there would a diminished capacity for the cells to perform this function.

When the external nutritional level matches the predicted nutritional level the foetuses' phenotypic alterations prove advantages and allow them to exist in an environment sparse in nutrition. However, when there are discrepancies between the prenatal and postnatal nutritional availability these metabolic adaptations become detrimental. It is this "mismatching" between the pre and postnatal environment which can lead to the increase in risk factors and the later development of disorders such as, NIDDM and cardiovascular disease [20]. 
The changes which occur in utero aim to maximise survival in adverse external environments. When postnatal nutrition is poor and energy expenditure is high these adaptations are advantageous. However, the somewhat recent rise of urbanisation has led to the development of obesogenic environments. There has been a shift in the global economic landscapes, an increasing number of countries are becoming more economically developed. As countries undergo economic development there is an increase in the availability of high calorie, high fat and high sugar foods and the promotion of sedentary lifestyles. As countries become more economically developed there is a decrease in overall energy expenditure, i.e. a decrease in the number of manual labour jobs. Functional adaptations such as the thrifty phenotype and predictive adaptive response become detrimental in these modern conditions; where postnatal nutrition is good and energy expenditure is low [20]. When postnatal nutrition is good and energy expenditure is low these adaptations for efficient energy storage and utilisation act to increase the risk of obesity and the risk of developing NIDDM later in life.

This is supported by the results gathered from the Hertfordshire cohort, the Dutch famine and the Helsinki study, when infants are born with low birth-weight but then go onto experience good postnatal nutrition they are prone to developing glucose intolerance and NIDDM. This phenomenon is most noticeable after the Dutch famine where, around one month after the liberation of the Netherlands from German occupation, the daily caloric intake rose swiftly to more than 2000 calories a day [5]. Creating an environment where early nutrition was bad and postnatal nutrition was good. Dramatically increasing the probability of an individual going on to develop NIDDM.

Adaptations that were once advantageous become detrimental in modern conditions. Those born with a low birth-weight have an increased ability to store nutrients efficiently and are more at risk of becoming obese in postnatal environments where energy expenditure is low, and nutrition is good. It is known that individuals born with low birth-weights have a reduced number of pancreatic beta cells, and obesity is a known risk factor for the development of NIDDM. A reduced number of beta cells combined with obesity in adulthood act to increase the risk of developing NIDDM exponentially.

\section{Proposed mechanisms}

The FOAD hypothesis has been well documented over the last 30 years; however, the mechanisms through which it acts still remain unclear. As the field of epigenetics and nutrigenomics has advanced it has become possible to explore potential mechanisms involved in alterations to foetal DNA, leading to increased risk of disease.

There are a number of different proposed mechanisms to explain the FOAD hypothesis. The "Predicted Adaptive Response" hypothesis suggests that the environment in utero is involved in "programming" the foetus in preparation for a similar postnatal environment e.g. one sparse in nutrition [7].

During this time epigenetic changes can occur acting to alter phenotypic adaptations above the level of the DNA. Epigenetic alterations to gene expression are reversible and to not change nucleotide sequences, nonetheless they are capable of being passed onto future generations. This was observed following the events of the Dutch Famine during World War 2. Where it was found that individuals exposed to the famine during the first two trimesters of their own development then went on to give birth to lower weight babies themselves, when compared to women who were not exposed to famine during their own development [19].
There are a number of mechanisms through which epigenetic changes to gene expression can occur. One such way is through chromatin modification; the function of chromatin is in the packaging of DNA and it is involved in the regulation of gene expression [32]. It has also been shown that variation in both histones and chromatin DNA can impact structure and expression of genes. Histones are proteins found in the cells of eukaryotes, and their function is to package DNA molecules into nucleosomes. Histones are able to undergo epigenetic modification through both acetylation and deacetylation. Acetylation of histones refers to the process whereby acetyl functional groups $\left(\mathrm{CH}_{3} \mathrm{CO}\right)$ becomes incorporated into the structure of the histone, deacetylation is the removal of this functional group. The addition of acetyl functional groups can act to make the chromatin less condensed allowing DNA transcription to take place [33]. Deacetylation triggers the opposite response and DNA transcription is unable to take place. The enzyme responsible for removing acetyl functional groups is histone deacetylase (HDAC) [33].

Another way through which the foetal genome could undergo alteration is through DNA methylation. This is the process through which a methyl group $\left(\mathrm{CH}_{3}\right)$ is added to the DNA molecule, which can lead to changes in the functional properties of the DNA molecule without altering the base pair sequence. The methyl group consistently attach to sections of DNA where a cytosine molecule is followed by a guanine molecule [33]. This reaction is catalysed by the enzymes methyltransferases, and the addition of a methyl group results in the target gene not being expressed [33].

It has been found that increased levels of circulating glucocorticoids are capable of leading to reduced rates of pancreatic beta cell formation [29]. Glucocorticoids are a class of steroid hormones, with one example being cortisol. It has been suggested that mothers who have experienced undernutrition during gestation have a reduced capacity for the production of placental $11 \beta$-Hydroxysteroid dehydrogenase, a group of enzymes capable of catalysing the reversible reaction of cortisol to cortisone and vice versa. The normal function of placental $11 \beta$-Hydroxysteroid dehydrogenase is in the prevention of active glucocorticoids from entering the placenta [34]. A reduction in function of placental $11 \beta$-Hydroxysteroid dehydrogenase would mean that the foetus of individuals experiencing undernutrition during pregnancy would be exposed to increased levels of active glucocorticoids, which has been shown to increase rates of reduced pancreatic beta cell formation.

Vitamin $\mathrm{A}$ is known to be involved in gene transcription, through binding to and regulating target genes. Rodent studies have demonstrated a moderate vitamin A deficiency can lead to disruptions to pancreatic beta cell growth. The potential involvement of vitamin $\mathrm{A}$ in regulation of gene expression and organ development should be considered in the future.

It is now generally accepted that the observed variation in phenotypic expression are the result of epigenetic alterations to the structure of the genome above the level of the DNA. A genotypes capacity to produce alternative forms of structure, physiological state or behaviour in response to environmental conditions is called 'developmental plasticity'. The mechanisms resulting in infants born with low birth-weights are also thought to be involved with the alterations to foetal programming that lead to the increased likelihood of these individuals developing chronic illness, such as NIDDM, in adult life. 


\section{Twin pregnancies}

In the UK approximately 1 in 65 births are multiple births [35]. Although in all of the experimental studies considered pregnancies were singleton births. In twin or multiple births, the foetuses are competing for both space and nutrients which may lead to their nutritional needs not being adequately met. Which in turn could lead to alterations in physiology in order to minimise short term negative repercussions. In the past it has been thought that the higher incidence of NIDDM in twin births was due to shared genetic factors, however with the development of the thrifty gene hypothesis it is now considered more likely that the higher rates of NIDDM in this group may be due to the shared early nutritional environment [22]. This observation is supported by another study conducted in twins, demonstrating that by the $7^{\text {th }}$ decade twins have increased incidents of insulin resistance and greater incidence of risk markers such as centripetal adiposity [11].

Those who have undergone fertility treatments such as in vitro fertilisation (IVF) are more likely to have multiple births. It is not uncommon for women who have undergo IVF to have had difficulty becoming pregnant naturally in the past. Factors that may alter fertility levels may also lead to increased risk of giving birth to low birth-weight babies. For example, older mothers ( $>35$ years) may have increased levels of DNA abnormalities associated with the aging process [2].

\section{Maternal overnutrition}

There is a global obesity epidemic, and according to the WHO, global obesity levels have increased nearly three times over in the past 4 decades [36]. As of 2016 it was estimated that there were over 1.9 billion adults ( $>18$ years old) who were classed as overweight, 650 million of whom were obese. An individual is classed as overweight if they have a BMI greater than or equal to $25 \mathrm{~kg} / \mathrm{m}^{2}$ and obese if their $\mathrm{BMI}$ is greater than or equal to $30 \mathrm{~kg} / \mathrm{m}^{2}$. Furthermore, the WHO reported that 350 million children and young people, aged between 5-19, were overweight or obese. Meaning the majority of the population now live in countries where more people die as a result of overnutrition as opposed to undernutrition.

As previously discussed glucose is the primary substrate of a foetus during oxidative respiration. Glucose passes from the maternal bloodstream across the placenta by way of facilitated diffusion, and insulin functions as a passive regulator of foetal growth [11]. In instances of maternal overnutrition there may be a higher level of maternal circulating blood glucose, leading to heavier infants at birth [11]. Excessive foetal growth and large for gestational age babies are more at risk of developing disease such as NIDDM later in life. It has been shown that both maternal overnutrition and impaired maternal glucose tolerance can lead to reduced glucose tolerance in the offspring [11]. This is supported by a study performed in 2003 which measured insulin sensitivity and secretion in 15 individuals who were exposed to diabetic environments while in utero, in comparison to a control group. This study found that exposed individuals were at greater risk of developing not only impaired glucose tolerance but also deficient insulin secretory response in adult life [37]. The relative risk was greater than could be explained through solely genetic factors alone. The use of individuals with type one diabetic fathers as a control meant that genetic influences could be accounted for.

During later stage of pregnancy, a mother can be at risk of developing gestational diabetes as a result of increased insulin resistance. Individuals with a high BMI $(>30)$ are more at risk of developing gestational diabetes in the second half of pregnancy. Maternal insulin resistance leads to increased levels of circulating glucose, acting to increase the concentration gradient between the placenta and maternal blood supply. Increasing the rates of facilitated diffusion of glucose into the placenta to the foetus. Both maternal and gestational diabetes can lead to increased foetal growth rate and large for gestational age babies [11]. It has been demonstrated that glucose overexposure can lead to increased foetal growth. But also, alterations to pancreatic beta cell development, which can lead to impaired insulin secretions and reduced functional capacity in adult life. Reduction in the number of pancreatic beta cells is also seen in individuals who are exposed to maternal undernutrition during foetal development. Suggesting both maternal undernutrition and maternal overnutrition result in the same physiological manifestations in adult life.

Overnutrition can also lead to hyperlipidaemia which can cause proinflammatory pathways to be activated while in utero. Which has the potential to affect metabolic processes and mitochondrial function [38]. Overweight and obese individuals are more likely to develop gestational hypertension can lead to a reducing in uteroplacental flow, which in turn would lead to low birth-weight [2].

There has been some level of investigation into the effect of individual dietary components involved in maternal overnutrition. At present the majority of experimental data has been gathered using animal models. A number of independent studies involving rodents have examined the effect of maternal diets high in fat [39-41]. Each of these studies found that a high fat maternal diet was associated with increased insulin resistance in the offspring. While results from animal studies are insufficient grounds for nutrition recommendations for humans they do demonstrate the need for further examination into dietary composition in regard to maternal overnutrition.

As global obesity levels continue to rise incidence of maternal overnutrition is expected to rise also. Much of the research into maternal nutrition and its influence on foetal development has revolved around examining the effects of undernutrition on foetal development of adult diseases. As maternal overnutrition continues to increase it becomes more pressing to understand how exactly maternal overnutrition influences foetal growth and development. It is now understood that J-shaped curve relationship exists between birth-weight and adult risk of disease development. Creating a more complex picture, as both maternal undernutrition and maternal overnutrition can lead to the develop impaired glucose tolerance in later life. The influence of maternal overnutrition on foetal development of adult disease is an ongoing area of research, meaning it is difficult to comment on the distribution of the relative importance of maternal under versus overnutrition at this point.

\section{Future Directions}

In the time since the original publication of the FOAD hypothesis nearly 30 years ago the theory has become widely accepted within the scientific community and a number of independent studies carried out globally have produced results in line with those reported by Barker [42-44]. Prior to the development of the hypothesis it was believed that individuals who experience non-ideal nutritional environment in utero may continue to do so after birth; however, such claims have been found lacking [4]. The FOAD hypothesis has also been criticised because factors other than nutrition are capable of causing a low weight at birth, such as hypoxia and stress.

The association between birth-weight and adult disease development is well documented, however much of the early work in 
this field was observational epidemiology. As the field has advanced there are a number of different proposed mechanisms in order to explain the FOAD hypothesis. However, the exact mechanisms through which it acts still remain unclear and must be established before large scale public health campaigns can be developed.

There is a global NIDDM epidemic, through better understanding of the foetal origins of this disease it would be possible to aid in the development of prophylactic health care measures. There are a number of ways in which the principles of the FOAD hypothesis can be integrated into current health care practice. The use of primary prevention techniques should be the focus, as it may result in more effective prevention. Maternal health both pre and post conception have been shown to have the capacity to alter the foetal growth trajectory. Meaning it is essential for intervention programs in a primary care setting to target specific groups who are at risk of poor maternal nutrition. People in less economically developed countries, single parents and teenage pregnancy present the greatest risk of poor maternal nutrition and would benefit the most from more developed maternal nutritional recommendations. This could be achieved by providing a more comprehensive nutrition education to these target groups, focusing on nutrients which have been identified in this review. As well as identifying potential barriers to healthy eating and overall health and wellbeing. Through targeting specific at-risk groups, it may be possible to develop more sophisticated intervention programs which would hopefully have high compliance rates. In general, during pregnancy individuals become more receptive to healthcare advice and guidelines, which should be capitalised upon.

Modern western society is obesogenic, promoting high calorie diets and low energy expenditure. The FOAD hypothesis explains the role of prenatal nutrition in adult disease development, and its role in increasing the risk of NIDDM and other diseases. The diseases which develop as a result of poor maternal nutrition also develop as a result high calorie diet and lack of physical activity. It may be more prudent to focus on developing public health campaigns which focus on combating all cause NIDDM rather than that stemming from physiological changes occurring during foetal development [45-47].

\section{Conclusion}

The original FOAD hypothesis was put forward by Dr Barker in 1990 , suggesting that alterations to the quantity or quality of maternal nutrition can lead to a reduction in weight at birth. And individuals born with a low birth-weight, $<2.5 \mathrm{~kg}$, were more likely to go on to develop chronic non-communicable diseases such as CVD and NIDDM in adulthood. In the time since the publication, this theory has become well established and generally accepted within the scientific community. Landmark studies in this area include the Hertfordshire cohort, the Helsinki study and the Dutch famine. These studies have aided the establishment of the importance of good maternal nutritional status during pregnancy and its relation between the adult health of the offspring.

The event during development which result in diminished foetal growth are multifactorial. A foetus's maximal growth potential is determined genetically, however factors occurring in utero can influence the actual weight at birth of the offspring. Many of the factors which lead to poor maternal nutritional status are the result of poor education or poverty. Through reducing these socio-economic factors, it would be possible to reduce the health care inequalities globally. It is now understood that it is not solely events occurring during gestation which is capable of influencing foetal growth trajectory, increasingly the role of preconceptional nutrition is being considered. With events occurring during the preconceptional period able to trigger epigenetic changes, above the level of the DNA, which can then be passed onto offspring.

The various roles of both macro and micronutrients in foetal develop have been discussed. However, the majority of research regarding the role of micronutrients in foetal development have been conducted in rodents. At present it is imperative that the potential role of micronutrients supplementation as a prophylactic approach to prevent low birth-weight be more thoroughly explored.

Regardless of the cause, low birth-weight is associated with alterations of the endocrine system. Manifesting particularly as a reduced number of insulin producing pancreatic beta cells. Reduction in beta cell function can lead to reduced functional capacity for insulin production, increasing the risk of developing NIDDM later in life. It has been found that the risk of developing adult onset NIDDM is greatest in individuals born with a low birth-weight but who become obese in adult life.

The FOAD hypothesis has been well documented; however, the mechanisms through which it acts still remain unclear. A better understanding the mechanisms involved in the foetal developmental origins of NIDDM wound allow for the identification of at risk individuals, and the evolution of new methods for tracing risk factors and pre-empting disease such as NIDDM. Global incidence and prevalence of NIDDM is increasing rapidly, this growing problem presents significant cost to, not only the individuals themselves, but to the wider society and health care structures.

In the past the FOAD hypothesis primarily focuses on the role of undernutrition in foetal development of adult diseases. However, as global obesity increases it would be logical to expect that disease states which develop as a result of maternal overnutrition to rise also. Increasing precedence for the understanding of the role of maternal overnutrition on adult onset of disease in the foetus. It is now understood that J-shaped curve relationship has been reported between birthweight and adult risk of disease development. Creating a more complex picture, as both maternal undernutrition and maternal overnutrition can lead to the development of impaired glucose tolerance in later life.

The existing body of evidence regarding the role of adaptive changes occurring during foetal development in relation to the development of NIDDM in adulthood has been reviewed. Perturbations leading to adaptive changes to foetal growth are multifactorial, extending beyond events solely occurring during gestation. And through the establishment of intervention campaigns aimed at improving overall nutritional status of reproductive aged women it would be possible to improve the health span of potential offspring. These campaigns should focus on targeting women from poorer socio-economic backgrounds who are the most at risk of poor nutrition, and most at risk of giving birth to low birth-weight babies.

\section{References}

1. Barker DJ (1990) The fetal and infant origins of adult disease. BMJ 301: 1111.

2. Safizadeh H, Momeni M, Danaei M, Kermani A, Bakhshandeh M, et al. (2017) Prevalence and risk factors of low birth weight in the Southeast of Iran. Int $J$ Prev Med 8: 12.

3. Barker DJ (2004) The developmental origins of chronic adult disease. Acta Paediatr Suppl 93: 26-33.

4. Barker D, Osmond C, Kajantie E, Eriksson J (2009) Growth and chronic disease: findings in the Helsinki Birth Cohort. Ann Hum Biol 36: 445-458. 
Citation: Stephenson S, Cunliffe A (2018) Foetal Developmental Origins of Adult Onset Non-Insulin Dependent Diabetes Mellitus (NIDDM). J Nutr Food Sci 8: 733. doi: 10.4172/2155-9600.1000733

Page 11 of 11

5. Roseboom T, de Rooij S, Painter R (2006) The Dutch famine and its long-term consequences for adult health. Early Hum Dev 82: 485-491.

6. Calkins K, Devaskar S (2011) Fetal origins of adult disease. Curr Probl Pediatr Adolesc Health Care 41: 158-176.

7. Bateson $P$, Gluckman $P$, Hanson $M$ (2014) The biology of developmental plasticity and the Predictive Adaptive Response hypothesis. J Physiol 592: 2357-2368.

8. Yajnik C, Deshmukh U (2012) Fetal programming: maternal nutrition and role of one-carbon metabolism. Rev Endocr Metab Disord 13: 121-127.

9. http://www.who.int/en/news-room/fact-sheets/detail/diabetes

10. Godfrey K, Barker D (2001) Fetal programming and adult health. Public Health Nutr 4: 611-624.

11. Bloomfield F, Spiroski A, Harding J (2013) Fetal growth factors and fetal nutrition. Semin Fetal Neonatal Med 18: 118-123.

12. Lacasse A, Rey E, Ferreira E, Morin C, Bérard A (2009) Epidemiology of nausea and vomiting of pregnancy: prevalence, severity, determinants, and the importance of race/ethnicity. BMC Pregnancy Childbirth 9: 26.

13. Watkins A, Ursell E, Panton R, Papenbrock T, Hollis L, et al. (2008) Adaptive responses by mouse early embryos to maternal diet protect fetal growth but predispose to adult onset disease. Biol Reprod 78: 299-306.

14. Kwong W (2000) Maternal undernutrition during the preimplantation period of rat development causes blastocyst abnormalities and programming of postnatal hypertension. Development 127: 4195-4202.

15. Cetin I, Alvino G, Radaelli T, Pardi G (2007) Fetal nutrition: a review. Acta Paediatr Suppl 94: 7-13.

16. Lumey L (1998) Compensatory placental growth after restricted maternal nutrition in early pregnancy. Placenta 19: 105-111.

17. Godfrey K, Barker D (2000) Fetal nutrition and adult disease. Am J Clin Nutr 71: 1344 S-1352S

18. King J, Butte N, Chez R, Haas J, Kleinman J, et al. (1990) Nutrition during pregnancy: executive summary by the subcommittee on nutritional status and weight gain during pregnancy and the subcommittee on dietary intake and nutrient supplements during pregnancy. Nutr Today 25: 13-22.

19. Lumey $L$ (1992) Decreased birthweights in infants after maternal in utero exposure to the Dutch famine of 1944-1945. Paediatr Perinat Epidemiol 6 : 240-253.

20. Muehlenbein $M(2010)$ Human evolutionary biology (1 ${ }^{\text {st }}$ Edn). New York: Cambridge University Press, p: 603

21. Godfrey K, Robinson S, Barker D, Osmond C, Cox V (1996) Maternal nutrition in early and late pregnancy in relation to placental and fetal growth. BMJ 312 : $410-410$.

22. Hales C, Barker D (1992) Type 2 (non-insulin-dependent) diabetes mellitus: the thrifty phenotype hypothesis. Diabetologia 35: 595-601.

23. Cogswell M, Parvanta I, Ickes L, Yip R, Brittenham G (2003) Iron supplementation during pregnancy, anemia, and birth weight: a randomized controlled trial. Am J Clin Nutr 78: 773-781.

24. Hurrell R, Egli I (2010) Iron bioavailability and dietary reference values. Am J Clin Nutr 91: 1461S-1467S.

25. Matthews K, Rhoten W, Driscoll H, Chertow B (2004) Vitamin A deficiency impairs fetal islet development and causes subsequent glucose intolerance in adult rats. J Nutr 134: 1958-1963.

26. Wu G, Imhoff-Kunsch B, Girard A (2012) Biological mechanisms for nutritional regulation of maternal health and fetal development. Paediatr Perinat Epidemiol 26: 4-26
27. Christian P, Stewart C (2010) Maternal micronutrient deficiency, fetal development, and the risk of chronic disease. J Nutr 140: 437-445.

28. McMillen I, MacLaughlin S, Muhlhausler B, Gentili S, Duffield J, et al. (2008) Developmental origins of adult health and disease: the role of periconceptional and foetal nutrition. Basic Clin Pharmacol Toxicol 102: 82-89.

29. Blondeau B, Lesage J, Czernichow P, Dupouy J, Bréant B (2001) Glucocorticoids impair fetal $\beta$-cell development in rats. Am J Physiol Endocrinol Metab 281: E592-E599.

30. Watkins W, Kotecha S, Kotecha S (2016) All-cause mortality of low birthweight infants in infancy, childhood, and adolescence: population study of England and Wales. PLoS Med 13: e1002018.

31. Victora C, Barros F, Horta B, Martorell R (2001) Short-term benefits of catchup growth for small-for-gestational-age infants. Int J Epidemiol 30: 1325-1330.

32. Campbell N, Reece J, Urry L, Cain ML, Wasserman SA, et al. (2014) Biology: a global approach (10 $10^{\text {th }}$ Edn). Harlow: Pearson, p: 1512.

33. Burrows C, Faudemer K, Ward R, Lindle C, Marshall R, et al. (n.d.) A-Leve year 2 biology ( $\left.1^{\text {st }} \mathrm{Edn}\right)$.

34. Langley-Evans $S$ (n.d.). Nutrition health and disease: a lifespan approach (2 Edn). WILEY Blackwell, p: 833.

35. https://www.nhs.uk/conditions/pregnancy-and-baby/what-causes-twins/

36. http://www.who.int/en/news-room/fact-sheets/detail/obesity-and-overweight

37. Sobngwi E, Boudou P, Mauvais-Jarvis F, Leblanc H, Velho G, et al. (2003) Effect of a diabetic environment in utero on predisposition to type 2 diabetes. Lancet 361: 1861-1865.

38. Heerwagen M, Miller M, Barbour L, Friedman J (2010) Maternal obesity and fetal metabolic programming: a fertile epigenetic soil. Am J Physiol Regul Integr Comp Physiol 299: R711-R722.

39. Armitage J, Taylor P, Poston L (2005) Experimental models of developmenta programming: consequences of exposure to an energy rich diet during development. J Physiol 565: 3-8.

40. Chen H, Simar D, Lambert K, Mercier J, Morris M (2008) Maternal and postnata overnutrition differentially impact appetite regulators and fuel metabolism. Endocrinology 149: 5348-5356.

41. Lucas E, Finn S, Cox A, Lock F, Watkins A (2009) The impact of maternal high fat nutrition on the next generation: food for thought? J Physiol 587: 3425-3426.

42. Stein C, Fall C, Kumaran K, Osmond C, Barker D, et al. (1996) Fetal growth and coronary heart disease in South India. Lancet 348: 1269-1273.

43. Leon D, Lithell H, Vagero D, Koupilova I, Mohsen R, et al. (1998) Reduced fetal growth rate and increased risk of death from ischaemic heart disease: cohor study of 15000 Swedish men and women born 1915-29. BMJ 317: 241-245.

44. Lithell H, McKeigue P, Berglund L, Mohsen R, Lithell U, et al. (1996) Relation of size at birth to non-insulin dependent diabetes and insulin concentrations in men aged 50-60 years. BMJ 312: 406-410.

45. Bakker R, Steegers E, Obradov A, Raat H, Hofman A, et al. (2010) Materna caffeine intake from coffee and tea, fetal growth, and the risks of adverse birth outcomes: the Generation R Study. Am J Clin Nutr 91: 1691-1698.

46. Bech B, Obel C, Henriksen T, Olsen J (2007) Effect of reducing caffeine intake on birth weight and length of gestation: randomised controlled trial. BMJ 334: 409.

47. Sengpiel V, Elind E, Bacelis J, Nilsson S, Grove J, et al. (2013) Materna caffeine intake during pregnancy is associated with birth weight but not with gestational length: results from a large prospective observational cohort study. BMC Med 11: 42 